Publ. RIMS, Kyoto, Univ.

12 Suppl. (1977), 141-146.

\title{
Micro-Analytic Structure of the S-Matrix and Related Functions
}

\author{
by \\ Masaki KAshiWARA, ${ }^{*(1)}$ Takahiro KAWAI**(2) \\ and Henry P. STAPP***(3)
}

In this report we summalize some recent results and make a conjecture on the micro-analytic structure of the $S$-matrix and related functions. The details of this report shall appear elsewhere. We refer the reader to Kawai-Stapp [4], [5] for the definition of the symbols and terminologies used here.

In order to determine the micro-analytic structure of the $S$-matrix and other bubble diagram functions we first examine the singularity spectrum of the phase space integral $I_{D}(p)=I_{D}\left(p_{1}, \cdots, p_{n}\right)$ associated with a (stable particle) Landau diagram $D$ with $n$ external lines, $N$ internal lines and $n^{\prime}$ vertices. A formal definition of the phase space integral $I_{D}(p)$ is given as follows:

$$
\begin{gathered}
\prod_{r=1}^{n} \delta\left(p_{r}{ }^{2}-\mu_{r}^{2}\right) Y\left(p_{r}^{0}\right) \int \prod_{j=1}^{n^{\prime}} \delta^{4}\left(\sum_{r=1}^{n}[j: r] p_{r}+\sum_{l=1}^{N}[j: l] k_{l}\right) \times \\
\times \prod_{l=1}^{N} \delta\left(k_{l}^{2}-m_{l}^{2}\right) Y\left(k_{l}^{0}\right) \prod_{l=1}^{N} d^{4} k_{l}
\end{gathered}
$$

where $Y$ is the Heaviside function.

Since the general rule for the operations on hyperfunctions ( $[7]$ Chapter I § 2) cannot be applied to (1) at $u=0$ points ([4], [5]), we first investigate the micro-analytic structure of the generalized phase space

Received September 8, 1976.

* Mathematical Institute, Nagoya University and Department of Mathematics, Harvard University.

** Research Institute for Mathematical Sciences, Kyoto University and Department of Mathematics, University of California, Berkeley.

*** Lawrence Berkeley Laboratory, University of California, Berkeley.

(1) Supported by National Science Foundation.

(2) Supported by Miller Institute for Basic Research in Science.

(3) Supported by ERDA. 
integral $I_{D}\left(p ; \lambda, \lambda^{\prime}\right)$ given by (2) and then apply the celebrated method of Hadamard-Riesz to study $I_{D}(p)$ by analytic continuation with respect to the parameters $\left(\lambda, \lambda^{\prime}\right)$ :

$$
\begin{gathered}
\prod_{r=1}^{n} \frac{\left(p_{r}{ }^{2}-\mu_{r}{ }^{2}\right)_{+}^{\lambda_{r}}}{\Gamma\left(\lambda_{r}+1\right)} Y\left(p_{r}{ }^{0}\right) \int \prod_{j=1}^{n^{\prime}} \delta^{4}\left(\sum_{r=1}^{n}[j: r] p_{r}+\sum_{l=1}^{N}[j: l] k_{l}\right) \times \\
\times \prod_{l=1}^{N} \frac{\left(k_{l}{ }^{2}-m_{l}{ }^{2}\right)_{+}^{\lambda_{l}{ }^{\prime}}}{\Gamma\left(\lambda_{l}{ }^{\prime}+1\right)} Y\left(k_{l}{ }^{0}\right) \prod_{l=1}^{N} d^{4} k_{l} .
\end{gathered}
$$

This function is well-defined as a hyperfunction if $\operatorname{Re} \lambda_{r}, \operatorname{Re} \lambda_{l}{ }^{\prime} \gg 0$, and Lemma 2 of [2] can be used to determine maximum possible singularity spectrum there. Then, by a delicate choice of a path of analytic continuation in $\left(\lambda, \lambda^{\prime}\right)$-space, we find that (2) reduces to (1) when $\lambda_{r}=-1$ $(r=1, \cdots, n)$ and $\lambda_{l}{ }^{\prime}=-1 \quad(l=1, \cdots, N)$, provided this point is reached by a specific path. Here one should note that we do not need any extra $\gamma$-factor other than $1 / \Gamma\left(\lambda_{r}+1\right)$ and $1 / \Gamma\left(\lambda_{l}{ }^{\prime}+1\right)$. This makes a clear contrast to the procedure of analytic renormalization of Feynman intergrals ([8]). Lastly, by using a theorem on propagation of singularities ([7] Chapter III Theorem 2.2.8), we find that $I_{D}(p)$ is well-defined and its singularity spectrum is confined to the following set $\widetilde{L}(D)$ :

$\widetilde{L}(D)=\left\{(p ; \sqrt{-1} u) \in \sqrt{-1} S^{*}\left(\boldsymbol{R}^{4 n}\right) ;\right.$ there exist real four-vectors $k_{l}(l=1, \cdots, N)$ and sequences of real scalars $\alpha_{l}{ }^{(m)}(l=1, \cdots, N)$ and $\beta_{r}{ }^{(m)}$ $(r=1, \cdots, n)$ and real four-vectors $p_{r}{ }^{(m)}, u_{r}{ }^{(m)}(r=1, \cdots, n), k_{l}{ }^{(m)} \quad(l=1$, $\cdots, N)$ and $v_{j}^{(m)}\left(j=1, \cdots, n^{\prime}\right)$ which satisfy following relation (3)\}

$$
\begin{cases}p_{r}{ }^{(m)} \rightarrow p_{r} \quad \text { with } \quad p_{r}{ }^{0}>0, p_{r}{ }^{2}=\mu_{r}{ }^{2} & (r=1, \cdots, n) \\ u_{r}{ }^{(m)} \rightarrow u_{r} & (r=1, \cdots, n) \\ k_{l}{ }^{(m)} \rightarrow k_{l} \quad \text { with } \quad k_{l}{ }^{0}>0, k_{l}{ }^{2}=m_{l}{ }^{2} & (l=1, \cdots, N) \\ \sum_{r=1}^{n}[j: r] p_{r}{ }^{(m)}+\sum_{l=1}^{N}[j: l] k_{l}{ }^{(m)}=0 & \left(j=1, \cdots, n^{\prime}\right) \\ u_{r}{ }^{(m)}=-\sum_{j=1}^{n^{\prime}}[j: r]\left(v_{j}{ }^{(m)}-\beta_{r}{ }^{(m)} p_{r}{ }^{(m)}\right) & (r=1, \cdots, n) \\ \sum_{j=1}^{n^{\prime}}[j: l] v_{j}{ }^{(m)}-\alpha_{l}{ }^{(m)} k_{l}{ }^{(m)} \rightarrow 0 & (l=1, \cdots, N)\end{cases}
$$

Remark. At first sight, the condition on $\widetilde{\mathcal{L}}$ might seem to be too 
loose. However, Lemma 1 of [2] claims that $\widetilde{\mathcal{L}}$ is contained in the real locus of a Lagrangian variety. (See also [3].) Hence $\widetilde{L}$ is one of the best possible sets of the sort according to the philosophy of Professor Sato.

Next, we examine the full content of the macrocausality condition with the aid of a semi-classical model of the scattering process, and find, by essentially the same arguments as in [1], that the singularity spectrum of the $S$-matrix should be confined to the set $\bigcup_{D} \widetilde{\mathcal{L}}^{+}(D)$, where $\widetilde{\mathcal{L}}^{+}(D)$ is defined just as $\widetilde{\mathcal{L}}(D)$ but with the additional sign conditions $\alpha_{l}{ }^{(m)} \geqq 0$.

Thus we are led to propose the following postulate and conjecture for the singularity spectrum of the $S$-matrix and of bubble diagram functions:

POSTUlate. The singularity spectrum of the S-matrix $S(p)$ is confined to $\widetilde{\mathcal{L}}^{+}=\bigcup_{D} \widetilde{\mathcal{L}}^{+}(D)$.

CONJECTURE. The singularity spectrum of the bubble diagram function $F^{B}(p)$ is confined to the set $\widetilde{\mathcal{L}}^{B}=\bigcup_{D \in \bar{B}} \widetilde{\mathcal{L}}^{\sigma}(D)$. Here $\widetilde{\mathcal{L}}^{\sigma}(D)$ is defined analogously to $\widetilde{\mathcal{L}}^{+}(D)$, but with a sign condition $\sigma_{l} \alpha_{l}{ }^{(m)} \geqq 0$ for every signed internal line $l$ of $D \in \bar{B}$.

Remark. Since the off-shell $S$-matrix $\widetilde{S}(p)$ should satisfy

$$
\left(p_{r}^{2}-\mu_{r}^{2}\right) \widetilde{S}(p)=0, r=1, \cdots, n,
$$

it is easy to see by the theorem on the propagation of singularities ([7] Chapter III Theorem 2.1.7) that the singularity spectrum of the on-shell $S$-matrix is described by the same equations with the equivalence relation that $u \equiv u^{\prime}$ if and only if $u_{r}-u_{r}{ }^{\prime}=\beta_{r} p_{r}$ holds for some real scalar $\beta_{r}$ $(r=1, \cdots, n)$. Clearly this equivalence relation is consistent with (3. e). Since $S(p)$ should also satisfy

$$
\left(\sum_{j, r}[j: r] p_{r}\right) S(p)=0,
$$

the singularity spectrum of the scattering amplitude $S_{r}(p)=$ 
$=S(p) / \hat{O}^{4}\left(\sum_{j, r}[j: r] p_{r}\right)$ can be discussed by the same method on the reduced mass-shell variety

$$
\mathscr{M}_{r}=\left\{p \in \mathbb{R}^{4 n} ; \sum_{j, r}[j: r] p_{r}=0, p_{r}{ }^{2}=\mu_{r}^{2}(r=1, \cdots, n)\right\}
$$

outside $\mathscr{H}_{\mathrm{exc}}=\left\{p \in \mathscr{M}\right.$; all $p_{r}$ 's are parallel $\}$.

As we have discussed above, this conjecture for the bubble diagram functions has been verified for phase space integrals. Practically speaking, this result resolves many $u=0$ problems that arise in the derivation of discontinuity formulas from the unitarity and macrocausality properties of the $S$-matrix.

The micro-analyticity of the $S$-matrix described by $\widetilde{\mathcal{L}}$ should be taken into account in formulating Sato's conjecture, especially at the points far away from the physical region, as Sato [6] proposes. This comment applies also to off-shell amplitudes.

Finally, we discuss the relationship of $\widetilde{L}^{\sigma}$ to the ordinary Landau variety $\mathcal{L}^{\sigma}$.

Definition 1. (Landau equation) A set $\left(p_{1}, \cdots, p_{n} ; u_{1}, \cdots, u_{n}\right) \equiv(p ; u)$ consisting of $n$ real four-vectors $p_{r}$ and $n$ real four-vectors $u_{r}$ is said to be a solution of Landau equations corresponding to signed Landau diagram $D$ if and only if there are real scalars $\beta_{l,+}$ and $\beta_{l,-} \quad(l=1, \cdots, N)$ and $\beta_{r}(r=1, \cdots, n)$ and real four-vectors $k_{l}, \widehat{u}_{l}(l=1, \cdots, N)$ and $v_{j}(j=1, \cdots$, $n^{\prime}$ ) which satisfy the following:

$$
\left\{\begin{array}{lr}
p_{r}{ }^{2}=\mu_{r}{ }^{2}, p_{r}^{0}>0 & (r=1, \cdots, n) \\
k_{l}{ }^{2}=m_{l}{ }^{2}, k_{l}{ }^{0}>0 & (l=1, \cdots, N) \\
\sum_{r=1}^{n}[j: r] p_{r}+\sum_{l=1}^{N}[j: l] k_{l}=0 & \left(j=1, \cdots, n^{\prime}\right) \\
u_{r}=-[j(r): r]\left(v_{j(r)}-\beta_{r} p_{r}\right) & (r=1, \cdots, n) \\
\hat{u}_{l}=v_{j^{+}(l)}-\beta_{l,+} k_{l} & \\
=v_{j-(l)}-\beta_{l,-} k_{l} & (l=1, \cdots, N) \\
\sigma_{l}\left(\beta_{l,+}-\beta_{l,-}\right) \geqq 0 \quad \text { for every } & \text { signed line } l
\end{array}\right.
$$


The set $\left\{(p ; \sqrt{-1} u) \in \sqrt{-1} S^{*}\left(\mathscr{M}_{r}-\mathscr{M}_{\mathrm{exc}}\right) ;(p ; u)\right.$ satisfies equation (6) $\}$ is denoted by $\mathcal{L}^{\sigma}(D)$ and called the Landau variety associated with $D$.

Definition 2. (Trivial vertex) A vertex of a diagram representing a solution of the Landau equations is called a trivial vertex if and only if all (internal and external) lines that touch the vertex are parallel.

Definition 3. (Bounded vertex) A vertex of a diagram representing a solution of the Landau equations is called unbounded if and only if it can go to infinity with all the non-trivial vertices and other variables $p_{r}, k_{l}, u_{r}$, and $\hat{u}_{l}$ occurring in the solution kept fixed. A vertex that is not unbounded is bounded.

Definition 4. (Exceptional solution) A solution of the Landau equations is called exceptional if and only if all bounded vertices corresponding to this solution lie at a common point.

\section{Definition 5. (Generalized Landau equations)}

The generalized Landau equations consist of a set of alternative sets of equations. The first alternative set consists of the ordinary Landau equations. There is another alternative set for each exceptional solution of the ordinary Landau equations. This alternative set fixes the energymomentum vectors $p$ and $k$ to be the same as in the exceptional solution, but allows the vertices to be shifted by some real four-vectors $\eta_{j, r}$ and $\hat{\eta}_{j, l}$. In particular, the original Landau equations (6. d) and (6.e) are replaced by the following equations:

$$
\begin{cases}u_{r}=-[j(r): r]\left(\left(v_{j(r)}+\eta_{j, r}\right)-\beta_{r} p_{r}\right) \quad(r=1, \cdots, n) & (7 . \mathrm{d}) \\ \hat{u}_{l}=v_{j^{+}(l)}+\hat{\eta}_{j^{+}(l), l}-\beta_{l,+} k_{l} & (l=1, \cdots, N) \\ =v_{j^{-(l)}}+\hat{\eta}_{j-(l), l}-\beta_{l,-} k_{l} & (7 . \mathrm{e}) \\ \eta_{j, r}=0 \text { and } \hat{\eta}_{j, l}=0 \text { unless } j \in J_{0}, & \left(7 . \mathrm{g}^{\prime}\right) \\ \text { where } J_{0} \text { is the index set of unbounded vertices of the } \\ \text { associated exceptional solution, and }\end{cases}
$$




$$
\begin{gathered}
\sum_{r=1}^{n}[j: r]\left(p_{r}{ }^{0} \eta_{j, r}^{\nu}-p_{r}{ }^{\nu} \eta_{j, r}^{0}\right)+\sum_{l=1}^{N}[j: l]\left(k_{l}{ }^{0} \hat{\eta}_{j, l}^{\nu}-k_{l}{ }^{\nu} \hat{\eta}_{j, l}^{0}\right)=0 \\
\left(j=1, \cdots, n^{\prime}, \nu=1,2,3\right)
\end{gathered}
$$

Definition 6. (Generalized $u=0$ point) A point $p$ is called a generalized $u=0$ point of $\mathcal{L}^{\sigma}(D)$ if and only if it is a $u=0$ point of $\mathcal{L}^{\sigma}(D)$ and some $u=0$ solution $(p ; u)=(p ; 0)$ of the corresponding Landau equations is nonexceptional.

Theorem. Outside the set of generalized $u=0$ points the variety $\widetilde{\mathcal{L}}^{\sigma}(D)$ coincides with the variety defined by the generalized Landau equations.

Remark. The definitions of generalized Landau equations and the generalized $u=0$ points given in [4] should be replaced by the definitions given here.

\section{References}

[1] Iagolnitzer, D. and H. P. Stapp, Macroscopic causality and physical region analyticity in $S$-matrix theory, Commun. math. Phys. 14 (1969), 15-55.

[2] Kashiwara, M. and T. Kawai, On a conjecture of Regge and Sato on Feynman integrals, Proc. Japan Acad. 52 (1976), 161-164.

[3] - Holonomic systems of linear differential equations and Feynman integrals. This proceeding paper.

[4] Kawai, T. and H. P. Stapp, Micro-local study of the $S$-matrix singularity structure, Lecture Notes in Phys. No. 39, Springer (1975), 38-48.

[5] - Discontinuity formula and Sato's conjecture. This proceeding paper.

[6] Sato, M., Recent development in hyperfunction theory and its application to physics, Lecture Notes in Phys. No. 39, Springer (1975), 13-29.

[7] Sato, M., T. Kawai and M. Kashiwara, Microfunctions and pseudo-differential equations, Lecture Notes in Math. No. 287, Springer (1973), 265-529.

[8] Speer, E. R., Generalized Feynman Amplitudes. Princeton University Press (1969). 